\title{
Clinical significance of serum galactose-deficient immunoglobulin A1 for detection of recurrent immunoglobulin A nephropathy in kidney transplant recipients
}

\author{
Woo Yeong Park ${ }^{1,2,3}$, Yaerim Kim ${ }^{1,2}$, Jin Hyuk Paek ${ }^{1,2}$, Kyubok Jin ${ }^{1,2}$, Seungyeup Han ${ }^{1,2}$ \\ ${ }^{1}$ Division of Nephrology, Department of Internal Medicine, Keimyung University School of Medicine, Daegu, Republic of Korea \\ ${ }^{2}$ Keimyung University Kidney Institute, Daegu, Republic of Korea \\ ${ }^{3}$ Institute for Cancer Research, Keimyung University, Daegu, Republic of Korea
}

Background: Recurrent glomerulonephritis (GN) is a common cause of allograft loss in kidney transplantation (KT), the most frequent of which is immunoglobulin A (IgA) nephropathy (IgAN). Galactose-deficient lgA1 (Gd-lgA1) plays a major role in the pathophysiology of IgAN, but the association between Gd-lgA1 and recurrent IgAN in kidney transplant recipients (KTRs) is uncertain. We aimed to evaluate the efficacy of Gd-lgA1 for prediction of recurrent lgAN and graft and patient survival according to Gd-lgA1 level.

Methods: We enrolled 27 KTRs who underwent allograft biopsy between 2009 and 2016 and measured the serum Gd-lgA1 level of each KTR. We divided the patients into two groups: nonrecurrent IgAN (patients with IgAN prior to KT who were not diagnosed with recurrent IgAN) and recurrent lgAN (patients with IgAN prior to KT who were diagnosed with recurrent IgAN).

Results: The mean serum Gd-lgA1 level was significantly higher in the recurrent IgAN group than in the nonrecurrent IgAN group $(6,419 \pm 3,675 \mathrm{ng} / \mathrm{mL}$ vs. 3,381 $\pm 2,844 \mathrm{ng} / \mathrm{mL}, \mathrm{p}=0.02)$. The cutoff value of serum Gd-lgA1 in receiver operating characteristic curve analysis was $4,338 \mathrm{ng} / \mathrm{mL}$ (area under the curve, 0.76; 95\% confidence interval [Cl], 0.57-0.95, p=0.02). Serum Gd-lgA1 level was an independent factor for recurrent IgAN (odds ratio, 17.60; 95\% Cl, 1.33-233.03, $p=0.03$ ). There was no significant difference in graft or patient survival between the two groups.

Conclusion: Serum Gd-lgA1 can be used as a diagnostic biomarker for recurrent lgAN in KT.

Keywords: Glomerulonephritis, Graft survival, Immunoglobulin A, Kidney transplantation, Survival

Received: October 7, 2020; Revised: December 21, 2020; Accepted: December 28, 2020

Editor: Eun Hui Bae, Chonnam National University, Gwangju, Republic of Korea

Correspondence: Seungyeup Han

Division of Nephrology, Department of Internal Medicine, Keimyung University School of Medicine, Keimyung University Kidney Institute, 1035 Dalgubeol-daero, Dalseo-gu, Daegu 42601, Republic of Korea. E-mail: hansy@dsmc.or.kr

ORCID: https://orcid.org/0000-0002-7561-6534

Copyright (C) 2021 by The Korean Society of Nephrology

(a) This is an Open Access article distributed under the terms of the Creative Commons Attribution Non-Commercial and No Derivatives License (http:// creativecommons.org/licenses/by-nc-nd/4.0/) which permits unrestricted non-commercial use, distribution of the material without any modifications, and reproduction in any medium, provided the original works properly cited. 


\section{Introduction}

One of the most common causes of allograft kidney loss is recurrent glomerulonephritis (GN), and immunoglobulin A (IgA) nephropathy (IgAN) is the most common cause of recurrent GN after kidney transplantation (KT) [1,2]. The pathophysiology of IgAN is the most potent of the "four hits" hypotheses [3], and galactose-deficient IgAl (Gd-IgA1) plays a major role in the pathophysiology of IgAN [4]. The main mechanism of action of Gd-IgAl is deposition with an autoimmune IgG complex in the mesangium and endothelium, subsequently damaging those cells [4]. The same mechanism can be assumed for recurrent IgAN after KT, but recurrent $\mathrm{GN}$ because of immunosuppressive drug administration does not have the same mechanism of action as IgAN of the general population. Considering that Gd-IgAl can be measured in the laboratory, it can be used as a diagnostic and prognostic biomarker for IgAN, but it is unclear whether recurrent IgAN after KT is similarly observed based on this clinical pattern and whether de novo IgAN is observed. However, the association between Gd-IgAl and occurrence of recurrent IgAN in kidney transplant recipients (KTRs) is uncertain. Therefore, in this study, we aimed to investigate whether Gd-IgAl is effective as a diagnostic and prognostic marker for recurrent IgAN.

\section{Methods}

\section{Study design}

We enrolled 27 KTRs with stored samples in the Biobank of Keimyung University Dongsan Hospital and who underwent allograft biopsy between 2009 and 2016. There were 17 living donor KTRs (10 living-related donor KTRs and seven living-unrelated donor KTRs) and 10 deceased donor KTRs. The living-related donor KTRs comprised four parent-to-child and six sibling-to-sibling KTRs; two wife-to-husband KTRs were included in the living-unrelated donor KTRs. The patients were divided into the nonrecurrent IgAN group (patients with IgAN prior to KT who were not diagnosed with recurrent IgAN after KT $[\mathrm{n}=14]$ ) and the recurrent IgAN group (patients with IgAN prior to KT who were diagnosed with recurrent $\operatorname{IgAN}[\mathrm{n}=13]$ ). The nonrecurrent IgAN group included KTRs with other recurrent GN issues, acute and chronic rejection, and calcineurin inhibitor (CNI) toxicity. We evaluated the clinical characteris- tics of the study population, status of immunosuppression at diagnosis, change in allograft function, severity of proteinuria at diagnosis, and graft and patient survival.

\section{Demographic and clinical data}

We investigated the age of donors and recipients at diagnosis of recurrent IgAN, sex of donors and recipients, KT type, frequency of KT, dialysis type prior to KT, dialysis vintage, causes of end-stage renal disease (ESRD), number of human leukocyte antigen (HLA) mismatches, immunosuppressant for induction and maintenance treatment, previous biopsy-proven acute rejection (BPAR), severity of proteinuria at diagnosis of recurrent IgAN, panel reactive antibody (PRA) > $50 \%$, and donor-specific antibody positivity. Allograft biopsy was performed when allograft dysfunction (decreased estimated glomerular filtration rate [eGFR]) or persistent microscopic hematuria and proteinuria were observed. Allograft biopsy was analyzed using the Banff 2013 classification [5]. Allograft function was measured based on eGFR according to the guidelines of the Chronic Kidney Disease Epidemiology Collaboration within 1 month before diagnosis and at 12 months, 3 years, and 5 years after diagnosis [6]. Proteinuria was measured using the spot urine protein-to-creatinine ratio $(\mathrm{g} / \mathrm{g})$.

Serum Gd-IgAl level was measured using the Gd-IgAl assay kit (Immuno-Biological Laboratories Co., Ltd., Gunma, Japan) through the enzyme-linked immunosorbent assay [7]. The level of each serum Gd-IgAl was obtained based on a standard curve with an appropriate regression curve on each plot (four-parameter logistics; optical density, $450 \mathrm{~nm}$ ). The assay for Gd-IgAl was performed in triplicate.

The Institutional Review Board (IRB) of Keimyung University Dongsan Hospital approved this study (No. 201702-030). The requirement for informed consent was waived by the IRB because use of patient data for research, except identifying personal information, was explained to all donor families and all recipients before KT. Therefore, as a retrospective medical record study, this study did not contain any distinguishable personal information, except clinical process and outcome.

\section{Immunosuppression protocols}

We administered basiliximab (Simulect, $20 \mathrm{mg}$ on days 0 and 
4; Novartis, Basel, Switzerland) for KTRs with low immunologic risk and antithymocyte globulin (Thymoglobulin, $1.5 \mathrm{mg} /$ $\mathrm{kg}$ on day 0 and $1.0 \mathrm{mg} / \mathrm{kg}$ from day 1 to day 3; Genzyme, Cambridge, MA, USA) for KTRs with high immunologic risk as induction immunosuppressants. We administered cyclosporine (Sandimmune, $3 \mathrm{mg} / \mathrm{kg}$, twice a day; Novartis AG, Basel, Switzerland) or tacrolimus (Prograf, $0.05 \mathrm{mg} / \mathrm{kg}$, twice a day; Astellas Pharma Inc., Toyama, Japan) as a CNI, prednisolone (30 - 20 - 10 - 5 - $0 \mathrm{mg}$, once a day, on a stepdown regimen), and mycophenolate mofetil (CellCept, 750 or 1,000 $\mathrm{mg}$, twice a day for 1 month after KT and subsequent 500 mg, twice a day; Hoffmann-La Roche Inc., Nutley, NJ, USA) as maintenance immunosuppressants. The treatment protocol for recurrent IgAN was use of an angiotensin receptor blocker at diagnosis; if it elicited no response, then $0.25 \mathrm{mg} /$ $\mathrm{kg}$ per day of oral steroid was used.

\section{Statistical analyses}

Continuous variables were analyzed using the Mann-Whitney $\mathrm{U}$ test, and categorical variables were analyzed using the chi-square or Fisher exact test. Graft and patient survival rates were evaluated using the Kaplan-Meier analysis with log-rank test. Univariate and multivariate analyses with logistic regression analysis were performed to investigate the risk factors for development of recurrent IgAN. The p-values less than 0.05 were considered statistically significant. Statistical analysis was performed using the PASW Statistics (version 18.0; IBM Corp., Armonk, NY, USA).

\section{Results}

\section{Baseline characteristics of the study population}

The mean follow-up period for all patients was $147.5 \pm 83.6$ months. Recipient age at KT was significantly lower in the recurrent IgAN group than in the nonrecurrent IgAN group $(32.8 \pm 11.5$ years vs. $41.9 \pm 9.9$ years, $p=0.04)$, but there was no significant difference in recipient age at diagnosis. There was also no significant difference in donor age at KT between the two groups. There were no significant differences in rates of recipient and donor sex, PRA > 50\%, use of induction and maintenance immunosuppressants, or previous BPAR between the two groups. The proportion of living donor KT was higher in the recurrent IgAN group than in the nonrecurrent IgAN group. Moreover, the proportion of deceased donor KT was higher in the nonrecurrent IgAN group than in the recurrent IgAN group. The mean number of HLA mismatches was significantly lower in the recurrent IgAN group than in the nonrecurrent IgAN group $(2.6 \pm 1.9$ vs. $3.9 \pm 1.1, \mathrm{p}=0.05)$. There were no significant differences between the recurrent IgAN and nonrecurrent IgAN groups in the other clinical parameters (Table 1).

\section{Comparison of clinical and laboratory parameters according to recurrent IgA nephropathy}

The mean serum Gd-IgAl level was significantly higher in the recurrent IgAN group than in the nonrecurrent IgAN group $(6,419 \pm 3,675 \mathrm{ng} / \mathrm{mL}$ vs. $3,381 \pm 2,844 \mathrm{ng} / \mathrm{mL}, \mathrm{p}=$ $0.02)$. The mean time between KT and allograft biopsy was longer in the recurrent IgAN group than in the nonrecurrent IgAN group ( $108.9 \pm 83.3$ months vs. $61.7 \pm 69.2$ months $)$. The proportion of steroid use was significantly lower in the recurrent IgAN group than in the nonrecurrent IgAN group. Allograft function between diagnosis and 5 years after diagnosis and proteinuria at diagnosis did not differ between the two groups (Table 2).

\section{Receiver operating characteristic curve analysis}

The cutoff value of serum Gd-IgAl in receiver operating characteristic curve analysis was $4,338 \mathrm{ng} / \mathrm{mL}$ (area under the curve, 0.76 ; 95\% confidence interval [CI], 0.57-0.95; $\mathrm{p}=0.02$ ). The sensitivity and specificity were $76.9 \%$ and $78.6 \%$, respectively. The positive predictive value and negative predictive value were $76.1 \%$ and $78.6 \%$, respectively (Fig. $1 \mathrm{~A}$ ). There was no significant difference in death-censored graft survival rate after diagnosis of recurrent IgAN or other diseases according to GdIgA1 level (Fig. 1B).

Comparison of death-censored allograft survival and patient survival according to development of recurrent IgA nephropathy and factors related to development of recurrent IgA nephropathy

A total of 14 patients (51.9\%; six patients [22.2\%] in the recurrent IgAN group and eight patients [29.6\%] in the nonrecurrent IgAN group) experienced graft failure. The causes of graft failure were chronic rejection (three [50.0\%] and 
Table 1. Comparison of clinical and laboratory parameters according to recurrent IgAN

\begin{tabular}{|c|c|c|c|}
\hline Variable & Recurrent lgAN & Nonrecurrent IgAN & p-value \\
\hline No. of patients & 13 & 14 & \\
\hline Recipient age at KT (yr) & $32.8 \pm 11.5$ & $41.9 \pm 9.9$ & 0.04 \\
\hline Recipient sex, male:female & $6(46.2): 7(53.8)$ & 11 (78.6):3 (21.4) & 0.12 \\
\hline Donor age at KT (yr) & $34.3 \pm 10.7$ & $38.9 \pm 11.6$ & 0.31 \\
\hline Donor sex, male:female & 7 (53.8):6 (46.2) & $5(35.7): 9(64.3)$ & 0.45 \\
\hline Donor type, living:deceased & 11 (84.6):2 (15.4) & $6(42.9): 8(57.1)$ & 0.05 \\
\hline Living-related:living-unrelated & $8: 3$ & $4: 2$ & \\
\hline HLA mismatch $(n)$ & $2.6 \pm 1.9$ & $3.9 \pm 1.1$ & 0.05 \\
\hline PRA > 50\% & $2(15.4)$ & $5(35.7)$ & 0.65 \\
\hline DSA & $2(15.4)$ & $6(42.9)$ & 0.37 \\
\hline None & $6(46.2)$ & $4(28.6)$ & \\
\hline \multicolumn{4}{|l|}{ Maintenance immunosuppressant } \\
\hline Cyclosporine:tacrolimus & $4(30.8): 9(69.2)$ & $2(14.3): 12$ (85.7) & 0.39 \\
\hline
\end{tabular}

Data are expressed as number only, mean \pm standard deviation, or number (\%).

BPAR, biopsy-proven acute rejection; DSA, donor-specific antibody; HLA, human leukocyte antigen; IgAN, immunoglobulin A nephropathy; KT, kidney transplantation; PRA, panel reactive antibody.

Table 2. Comparison of clinical outcomes according to recurrent IgAN

\begin{tabular}{|c|c|c|c|}
\hline Variable & Recurrent IgAN $(n=13)$ & Nonrecurrent IgAN ( $n=14)$ & p-value \\
\hline Gd-lgA1 (ng/mL) & $6,418 \pm 3,675$ & $3,381 \pm 2,844$ & 0.02 \\
\hline Time from KT to biopsy (mo) & $108.9 \pm 83.3$ & $61.7 \pm 69.2$ & 0.12 \\
\hline \multicolumn{4}{|l|}{ Immunosuppressant at diagnosis } \\
\hline Cyclosporine:tacrolimus & $2(15.4): 11(84.6)$ & 2 (14.3):12 (85.7) & $>0.99$ \\
\hline Mycophenolate mofetil & $7(53.8)$ & $11(78.6)$ & 0.24 \\
\hline \multicolumn{4}{|l|}{ Steroid } \\
\hline After diagnosis & $8(61.5)$ & $8(57.1)$ & $>0.99$ \\
\hline \multicolumn{4}{|l|}{ ARB } \\
\hline Before diagnosis & $3(23.1)$ & $3(21.4)$ & $>0.99$ \\
\hline After diagnosis & $8(61.5)$ & $5(35.7)$ & 0.26 \\
\hline \multicolumn{4}{|c|}{ Allograft function (CKD-EPI) (mL/min/1.73 $\left.\mathrm{m}^{2}\right)$} \\
\hline 2 & $48.9 \pm 25.1$ & $43.3 \pm 22.8$ & 0.58 \\
\hline 3 & $51.1 \pm 16.8$ & $35.8 \pm 22.8$ & 0.09 \\
\hline 4 & $51.4 \pm 23.6$ & $43.9 \pm 29.6$ & 0.57 \\
\hline 5 & $44.4 \pm 23.5$ & $32.4 \pm 23.2$ & 0.35 \\
\hline Proteinuria at diagnosis (g/g) & $1.7 \pm 1.9$ & $1.0 \pm 0.8$ & 0.22 \\
\hline
\end{tabular}

Data are expressed as mean \pm standard deviation or number (\%).

ARB, angiotensin receptor blocker; CKD-EPI, Chronic Kidney Disease Epidemiology Collaboration; Gd-lgA1, galactose-deficient immunoglobulin A1; IgAN, immunoglobulin A nephropathy; KT, kidney transplantation. 

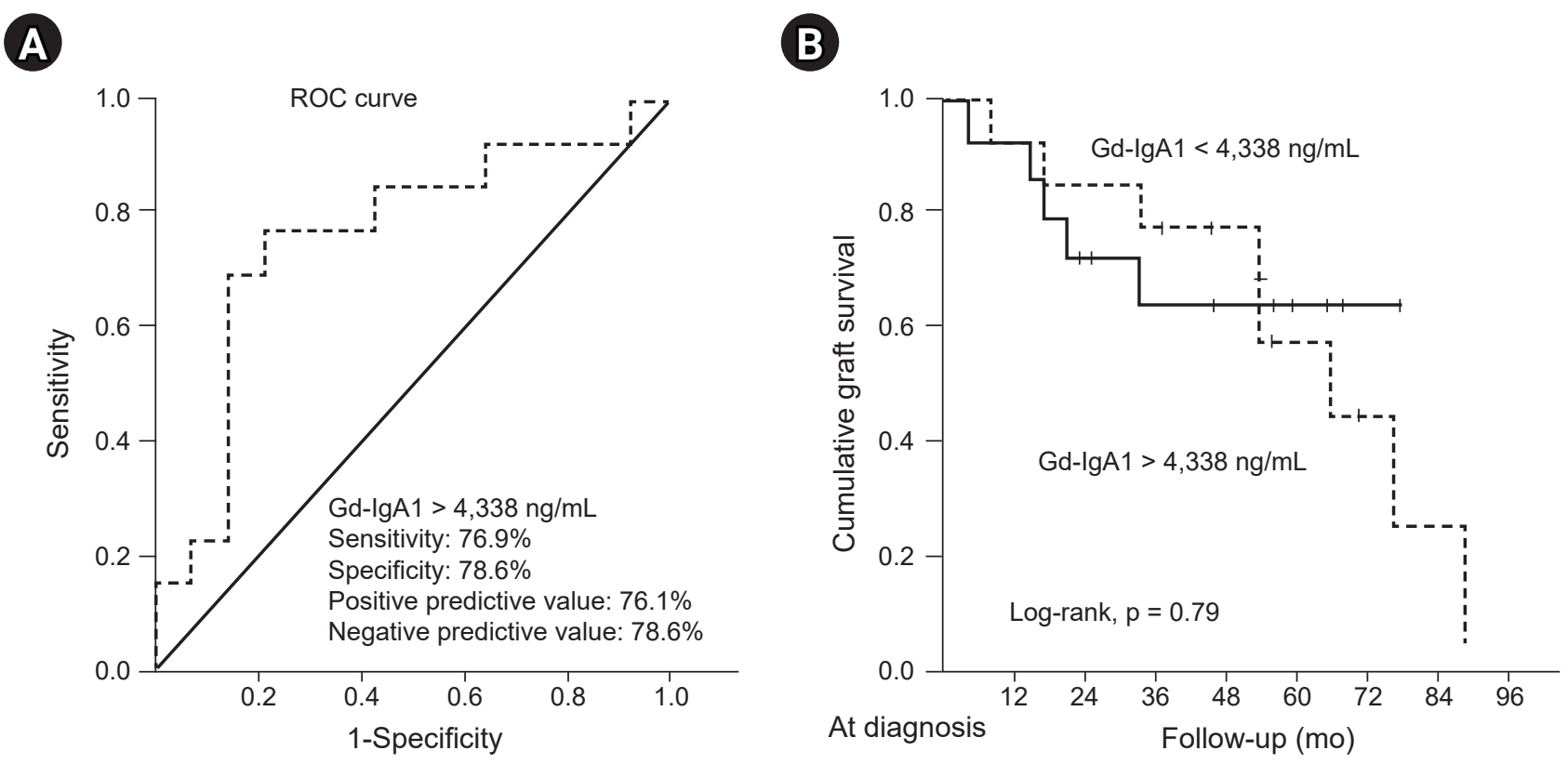

Figure 1. The cutoff of Gd-lgA1 level and allograft outcome according to the Gd-lgA1 level. (A) ROC curves for serum Gd-lgA1 level. ROC AUC for recurrence of IgAN was $0.76(0.57-0.95)$ for serum Gd-lgA1 ( $p=0.023)$. (B) Comparison of death-censored graft survival rate after diagnosis of recurrent IgAN according to Gd-lgA1 level.

AUC, area under the curve; Gd-IgA1, galactose-deficient-immunoglobulin A1; IgAN, immunoglobulin A nephropathy; ROC, receiver operating characteristic.

Table 3. Comparison of cause of allograft failure and patient death according to recurrent IgAN

\begin{tabular}{|c|c|c|c|}
\hline Variable & Recurrent IgAN $(n=13)$ & Nonrecurrent IgAN $(n=14)$ & p-value \\
\hline Causes of graft failure & & & 0.08 \\
\hline Chronic rejection & $3(23.1)$ & $6(42.9)$ & \\
\hline Recurrent IgAN & $3(23.1)$ & $0(0)$ & \\
\hline Others & $0(0)$ & $1(7.1)$ & \\
\hline Patient death with a functioning graft & $0(0)$ & $1(7.1)$ & \\
\hline Causes of death & & & $>0.99$ \\
\hline Cytomegalovirus pneumonia & $0(0)$ & $1(7.1)$ & \\
\hline
\end{tabular}

Data are expressed as number (\%).

IgAN, immunoglobulin A nephropathy.

six [75.0\%] in the recurrent IgAN and nonrecurrent IgAN groups, respectively), recurrent IgAN (three [50.0\%] and none in the recurrent IgAN and nonrecurrent IgAN groups, respectively), and patient death with graft function (none and one $[12.5 \%]$ in the recurrent IgAN and nonrecurrent IgAN groups, respectively) (Table 3 ). The 10-year graft survival rate was $88.9 \%$ in the recurrent IgAN group and $69.6 \%$ in the nonrecurrent IgAN group, but there was no significant difference in overall death-censored graft survival between the two groups (Fig. 2A). There was no significant difference in death-censored graft survival rate after diagnosis of re- current IgAN or other diseases between the two groups (Fig. 2B). One patient (3.7\%) in the nonrecurrent IgAN group died due to cytomegalovirus pneumonia. The 10-year patient survival rate was $100 \%$ in the recurrent IgA group and $92.9 \%$ in the nonrecurrent IgAN group, but there was no significant difference in death-censored graft survival between the two groups.

Based on logistic regression analysis, serum Gd-IgAl level was an independent factor for diagnosis of recurrent IgAN (odds ratio, 17.06; 95\% CI, 1.33-233.03; $\mathrm{p}=0.03$ ) adjusting for recipient age, sex, living donor $\mathrm{KT}$, dialysis vintage, mainte- 

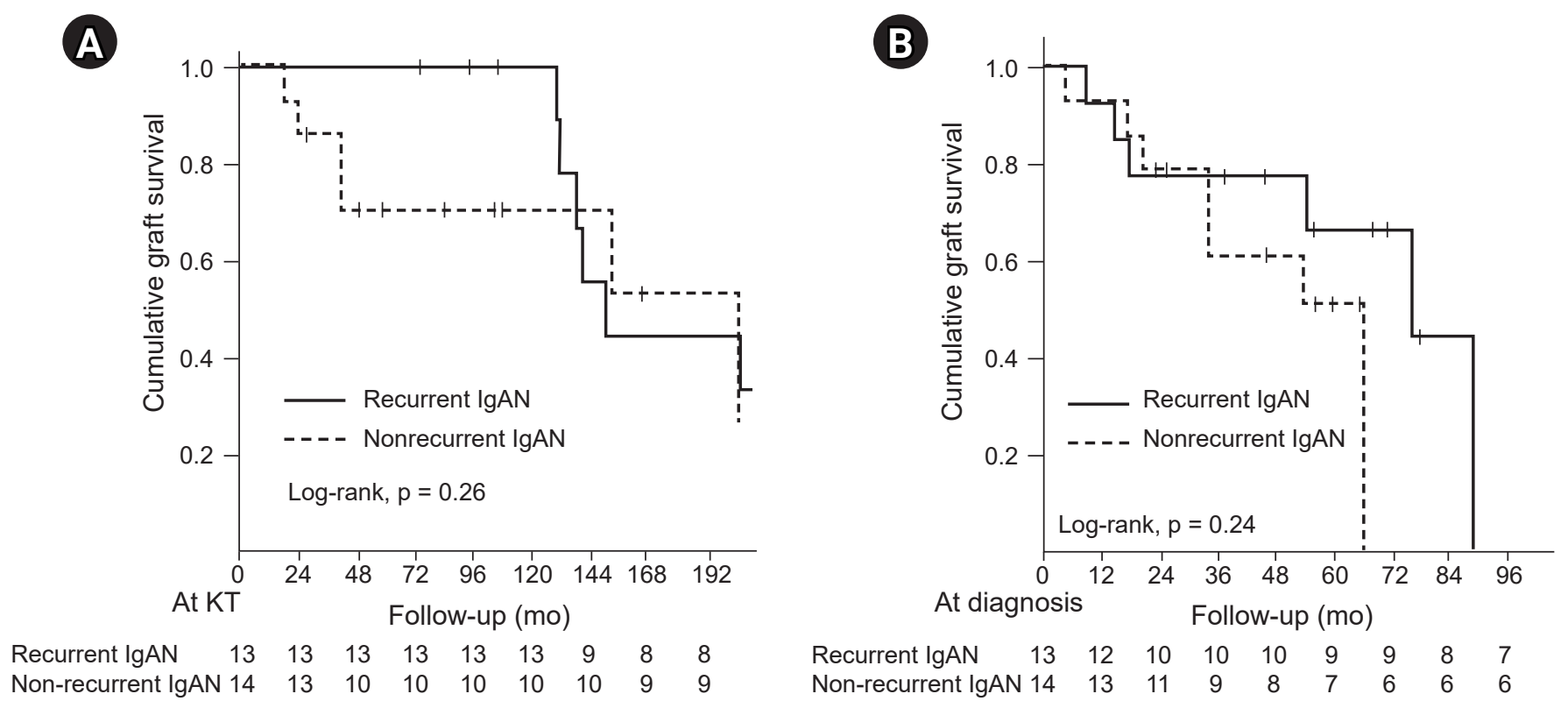

Figure 2. Allograft outcome of kidney transplant recipients according to the development of recurrent lgAN. Comparison of (A) deathcensored overall graft survival rate and $(B)$ death-censored graft survival rate after diagnosis of recurrent lgAN.

IgAN, immunoglobulin A nephropathy; KT, kidney transplantation.

Table 4. Risk factors associated with recurrent IgA nephropathy in KT recipients

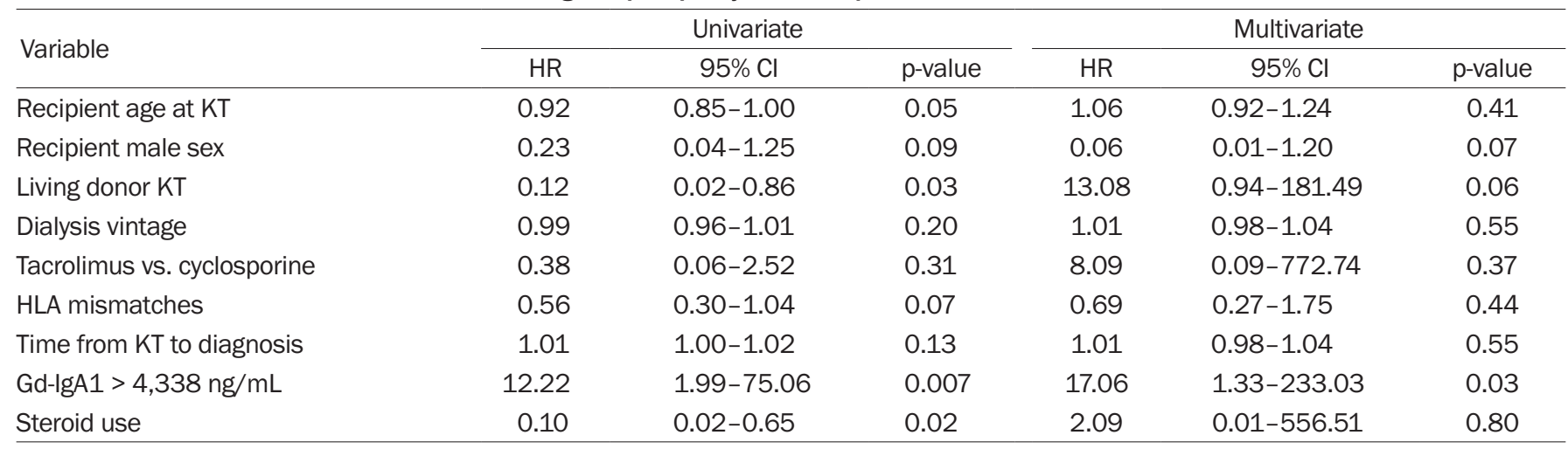

$\mathrm{Cl}$, confidence interval; Gd-lgA1, galactose-deficient-immunoglobulin A1; HLA, human leukocyte antigen; HR, hazard ratio; IgA, immunoglobulin A; KT, kidney transplantation.

nance immunosuppressant, HLA mismatch, time from KT to diagnosis, and steroid use (Table 4).

\section{Discussion}

IgAN has been implicated in the most frequent, primary form of GN and is the most common cause of recurrent GN in KTRs. However, the clinical manifestations of recurrent GN include proteinuria and allograft dysfunction such as acute rejection, recurrent GN, or CNI toxicity. To identify the causes of these symptoms, allograft biopsy is currently the best diagnostic method [8]. However, allograft biopsy is invasive, requires hospitalization, and can lead to false diagnosis due to insufficient specimens. Therefore, several studies are being conducted to overcome these shortcomings; in the case of recurrent IgAN, the research has been conducted to identify the mechanism of IgAN in native kidney diseases [9]. In particular, Gd-IgAl, involved in the mechanism of IgAN, has been reported as a diagnostic biomarker [10]. We evaluated the usefulness of Gd-IgAl as a diagnostic biomarker for recurrent IgAN in KTRs.

Temurhan et al. [11] assessed the Gd-IgAl level of recur- 
rent IgAN in KT, nonrecurrent IgAN in KT, non-transplant IgAN patients, and healthy relatives. They found that, regardless of transplantation, Gd-IgAl level was significantly higher in patients with recurrent IgAN and non-transplant IgAN than in those with nonrecurrent IgAN and healthy relatives (recurrent IgAN, 8,735 $\pm 10,854 \mathrm{ng} / \mathrm{mL}$; non-transplant IgAN, 8,791 \pm 8,700 ng/mL; nonrecurrent IgAN, 4,790 $\pm 6,089 \mathrm{ng} / \mathrm{mL}$; healthy relatives, $2,615 \pm 1,611 \mathrm{ng} / \mathrm{mL})$. The results of our study are consistent with those of the abovementioned study. Patients with recurrent IgAN after KT showed significantly higher Gd-IgAl level than patients with nonrecurrent IgAN after KT.

Because Gd-IgAl level is specifically elevated in IgAN patients according to pathogenesis of IgAN, IgAN can be predicted by Gd-IgAl level, although the cause of ESRD is not confirmed through kidney biopsy. In addition, in KTRs, it is possible to indirectly evaluate the status of the allograft kidney for presence of acute or chronic rejection or CNI toxicity, although the Gd-IgAl level is not elevated and the cause of ESRD is not IgAN, which can overcome the disadvantages of allograft biopsy.

In other words, when KTRs with IgAN as a cause of ESRD have allograft dysfunction or new-onset proteinuria or hematuria, we recommend measuring the Gd-IgAl level with blood samples. If the Gd-IgAl level is high, we can consider recurrent IgAN, which will lead us to use an angiotensin receptor blocker or steroid without allograft biopsy.

Interestingly, the proportion of living donor KT was higher in the recurrent IgAN group than in the nonrecurrent IgAN group. Some studies have reported that KT from a living-related donor is the most well-known risk factor for recurrent IgAN, and the incidence of IgAN in renal allografts is higher in living-related donor KT than in deceased donor KT. The results of our study are consistent with those of the abovementioned studies [12,13]. In our study, multivariate logistic regression analysis showed an odds ratio of living donor KT for occurrence of recurrent IgAN of 13.08 (95\% CI, 0.94181.49, $\mathrm{p}=0.055$ ); therefore, the occurrence of recurrent IgA is associated with living donor KT. However, there was no significant difference in allograft survival of patients with recurrent IgAN between living donor KT and deceased donor KT. These observations were not confirmed in other studies $[14,15]$, and there was no speculated reason for the higher recurrence rate of IgAN in living donor KT than in deceased donor KT. Therefore, further large-scale studies are needed.
There is a lack of consensus regarding the role of steroids against occurrence of recurrent IgAN. Allen et al. [16] reported that corticosteroids reduced the occurrence of recurrent IgAN in KTR. In our study, the proportion of steroid use at diagnosis was significantly lower in the recurrent IgAN group compared with the nonrecurrent IgAN group, but steroid use was not a factor related to development of recurrent IgAN in multivariate analysis. Furthermore, steroid was used when there was no response to angiotensin receptor blocker, and steroid was not effective in most patients with recurrent IgAN.

Our study has some limitations. First, this was a retrospective study. Therefore, since a selection bias can develop, further large-scale prospective studies are needed. Second, we did not assess Gd-IgAl level regularly after KT because examination for Gd-IgAl was not commercialized for research purposes. In other words, we only measured Gd-IgAl level at the time of allograft biopsy in blood samples stored in the Biobank for evaluation of allograft dysfunction. Third, because the sample size was small, the results of this study might not be representative. In particular, we cannot compare the difference in Gd-IgAl level according to the effect of induction immunosuppressant because of small sample sizes. However, we did observe significant differences in serum Gd-IgAl level that might indicate recurrent IgAN in KTRs with allograft dysfunction.

In conclusion, serum Gd-IgAl can be effective for early detection of recurrent IgAN in KTRs. Therefore, serum GdIgAl can be used as a diagnostic biomarker for recurrent IgAN in KT.

\section{Conflicts of interest}

All authors have no conflicts of interest to declare.

\section{Funding}

This study was supported by the Basic Science Research Program through the National Research Foundation of Korea (NRF) funded by the Ministry of Education, Science, and Technology (NRF-2017R1D1A1B03029140) and was also supported by the First Research Support Project of the National Research Foundation of Korea (NRF) funded by the Ministry of Science and ICT in 2018 (NRF-2017R1C1B5076739). 


\section{Authors' contributions}

Conceptualization, Project administration: $\mathrm{SH}$

Data curation, Funding acquisition: WYP

Investigation: YK, JHP, KJ

Writing-original draft: WYP

Writing-review \& editing: All authors

All authors read and approved the final manuscript.

\section{Acknowledgments}

The biospecimens and data used for this study were provided by the Keimyung University Dongsan Hospital Biobank a member of the Korea Biobank Network.

\section{ORCID}

Woo Yeong Park, https://orcid.org/0000-0003-2662-2898

Yaerim Kim, https://orcid.org/0000-0003-1596-1528

Jin Hyuk Paek, https://orcid.org/0000-0001-8875-1260

Kyubok Jin, https://orcid.org/0000-0002-7836-8863

Seungyeup Han, https://orcid.org/0000-0002-7561-6534

\section{References}

1. Wang AY, Lai FM, Yu AW, et al. Recurrent IgA nephropathy in renal transplant allografts. Am J Kidney Dis 2001;38:588-596.

2. An JN, Lee JP, Oh YJ, et al. Incidence of post-transplant glomerulonephritis and its impact on graft outcome. Kidney Res Clin Pract 2012;31:219226

3. Chang S, Li XK. The role of immune modulation in pathogenesis of IgA nephropathy. Front Med (Lausanne) 2020;7:92.

4. Floege J. Primary glomerulonephritis: a review of important recent discoveries. Kidney Res Clin Pract 2013;32:103-110.

5. Hara S. Banff 2013 update: pearls and pitfalls in transplant renal pathology. Nephrology (Carlton) 2015;20 Suppl 2:2-8.

6. Inker LA, Eckfeldt J, Levey AS, et al. Expressing the CKD-EPI (Chronic Kidney Disease Epidemiology Collaboration) cystatin C equations for estimating GFR with standardized serum cystatin C values. Am J Kidney Dis 2011;58:682-684.

7. Yasutake J, Suzuki Y, Suzuki H, et al. Novel lectin-independent approach to detect galactose-deficient IgAl in IgA nephropathy. Nephrol Dial Transplant 2015;30:1315-1321.

8. Al-Awwa IA, Hariharan S, First MR. Importance of allograft biopsy in renal transplant recipients: correlation between clinical and histological diagnosis. Am J Kidney Dis 1998;31:S15-S18.

9. Hastings MC, Moldoveanu Z, Suzuki H, et al. Biomarkers in IgA nephropathy: relationship to pathogenetic hits. Expert Opin Med Diagn 2013;7:615-627.

10. Berthelot L, Robert T, Vuiblet V, et al. Recurrent IgA nephropathy is predicted by altered glycosylated IgA, autoantibodies and soluble CD89 complexes. Kidney Int 2015;88:815-822.

11. Temurhan S, Akgul SU, Caliskan Y, et al. A novel biomarker for post-transplant recurrent IgA nephropathy. Transplant Proc 2017;49:541-545.

12. Deng R, Dai $Y$, Zhang $H$, et al. Higher incidence of renal allograft glomerulonephritis in living-related donor kidney transplantation. Transplant Proc 2018;50:2421-2425.

13. Han SS, Huh W, Park SK, et al. Impact of recurrent disease and chronic allograft nephropathy on the long-term allograft outcome in patients with IgA nephropathy. Transpl Int 2010;23:169-175.

14. Ponticelli C, Traversi L, Banfi G. Renal transplantation in patients with IgA mesangial glomerulonephritis. Pediatr Transplant 2004;8:334-338.

15. Cosio FG, Cattran DC. Recent advances in our understanding of recurrent primary glomerulonephritis after kidney transplantation. Kidney Int 2017;91:304-314.

16. Allen PJ, Chadban SJ, Craig JC, et al. Recurrent glomerulonephritis after kidney transplantation: risk factors and allograft outcomes. Kidney Int 2017;92:461-469. 